R. D. Davies. High-resolution studies at Jodrell Bank show the existence of neutral hydrogen in a ring of diameter $30^{\prime}$ surrounding the Orion Nebula. The mass of this material is $150 \odot$, and the density within the shell is $\mathrm{I}_{7}$ (neutral) $n_{\mathrm{H}} \mathrm{cm}^{-3}$.

$H$. van Woerden. There does appear to be neutral hydrogen close to the Nebula in space. It may be that the ionizing radiation is partly shielded by dense clumps or filaments of neutral $\mathrm{H}$, and that part of the radiation leaks out of holes between.

G. Münch. The real problem related to the age of the Orion Nebula is not how long it has been an expanding Hir region, but rather how a very massive complex with density exceeding $10^{4}$ atoms $\mathrm{cm}^{-3}$ managed to exist for the age of the Galaxy, notwithstanding the disruptive effects of tidal forces, pressure gradients, supernova explosions, and so forth. Or in other words, where in the sky is the complex which will produce a similar nebula to Orion about $10^{7}$ years from now?

\title{
10. INHOMOGENEITIES IN THE ORION NEBULA
}

\section{F. D. Kahn}

Determinations of the (electron) density in the Orion Nebula seem really to lead to an evaluation of $\left(\overline{n^{2}}\right)^{1 / 2}$, for any given region. In deducing $\bar{n}$ from $\left(\overline{n^{2}}\right)^{1 / 2}$, one usually forgets that there may be important small-scale fluctuations in the density $n$, and so one overestimates $\bar{n}$. Perhaps this is how the inference has arisen that there is such a violent density and pressure gradient in the Nebula, which then results in calculations such as Dr Vandervoort's in a short predicted lifetime. In reality, this short lifetime may simply be due to these being more pronounced density fluctuations near the centre of the Nebula. Dr Menon has already given reasons why these might have to be postulated in any case.

A possible origin of such fluctuations is as follows: consider a collection of globules of nonionized gas, each held together by self-gravitation. On exposure to Lyman continuum radiation, each globule surrounds itself with a bright rim of ionized gas, which expands outward. The incident radiation has to keep this gas ionized, and in this way the neutral gas is almost, but not entirely, shielded from the radiation. This may be expressed by the relation

$$
\mathcal{y} \cong \beta \int_{R}^{\infty} n^{2} d r
$$

where, for simplicity, spherical symmetry is assumed. The motion of the ionized gas is governed by the equation of continuity

and by Bernouilli's equation

$$
F=n u r^{2}
$$

$$
\frac{1}{2} u^{2}+a^{2} \log \frac{n}{n_{0}}=\frac{1}{2} a^{2}
$$

wherein

$\beta=$ the recombination coefficient $\left(\cong \mathrm{IO}^{-13} \mathrm{~cm}^{3} \mathrm{sec}^{-1}\right)$,

$\mathcal{f}=$ incident flux in the Lyman continuum ( $\cong{ }_{10}^{12}$ photons $\left.\mathrm{cm}^{-2} \mathrm{sec}^{-1}\right)$,

$a=$ isothermal speed of sound in the ionized gas $\left(\cong 10^{6} \mathrm{~cm} \mathrm{sec}^{-1}\right)$,

$n_{0}=$ density of ionized gas at ionization front,

$R=$ radius of ionization front. 
In equation (3) it has been assumed that the ionized gas streams away from the ionization front with the speed of sound, and that it experiences negligible gravitational effects. On setting

$$
n=n_{0} \exp (-\eta)
$$

one finds from (2) and (3) that the rate of loss of particles from the globule in terms of $\mathcal{F}$ is

$$
4 \pi F=\frac{64 \pi \mathcal{F}^{2} a}{\beta^{2} n_{0}^{3}} \cong \frac{2 \times 10^{58}}{n_{0}^{3}} \mathrm{sec}^{-1}
$$

with our assumed values. The corresponding rate of mass loss is about $4 \times 10^{34} / n_{0}^{3} \mathrm{gm} \mathrm{sec}^{-1}$; the lifetime per solar mass is then $n_{0}{ }^{3} / 20$ seconds (where, as in all these expressions, $n_{0}$ is in units of $\mathrm{cm}^{-3}$ ). Thus if $n_{0}=10^{5} \mathrm{~cm}^{-3}$, the lifetime of a globule is quite long as far as loss of matter by ionization is concerned.

The size of the typical condensation can be estimated as follows: The density drops to $10^{2}$ $\mathrm{cm}^{-3}$ when $\eta \cong 7$ (under our assumptions). We find then that $r \cong 6 \times 10^{16} \mathrm{~cm}$. Now the rim should begin to merge with the background at about this stage; the gas has reached a speed of $u=a(\mathrm{I}+2 \eta)^{\frac{1}{2}} \cong 40 \mathrm{~km} / \mathrm{sec}$ then.

Perhaps the interaction of gas flows, running from different globules at such speeds in different directions, may give an explanation of the velocity patterns described by Münch.

It is hoped soon to work out these ideas in greater detail.

\section{DISCUSSION}

$P$. Vandervoort. In reply to Dr Kahn, if the gas is much less concentrated to the centre than we have assumed, then it is difficult to understand why the dust is so centrally condensed. With the gas density distribution suggested by Dr Kahn, the ratio of dust to gas decreases outward, while the opposite might be expected if the dust tends to evaporate. Furthermore, any model requires only that the mean density and the r.m.s. density must be the same function of position, but they are allowed to differ by some factor. 